\title{
Provably secure certificateless encryption scheme in the standard model
}

\author{
Lunzhi Deng $^{1 *}$, Tian Xia ${ }^{1}$, Xiuru He ${ }^{1}$ \\ 1. School of Mathematical Sciences, Guizhou Normal University, Guiyang 550001, China. \\ *Corresponding author: Lunzhi Deng
}

Received August 30, 2019; revised March 16, 2020; accepted March 31, 2020;

published June 30, 2020

\begin{abstract}
Recently, numerous certificateless encryption (CLE) schemes have been introduced. The security proofs of most schemes are given under the random oracle model (ROM). In the standard model, the adversary is able to calculate the hash function instead of asking the challenger. Currently, there is only one scheme that was proved to be secure in SM. In this paper, we constructed a new CLE scheme and gave the security proofs in SM. In the new scheme, the size of the storage space required by the system is constant. The computation cost is lower than other CLE schemes due to it needs only two pairing operations.
\end{abstract}

Keywords: Certificateless encryption, Pairing, Standard model, Diffie-Hellman problem, Security 


\section{Introduction}

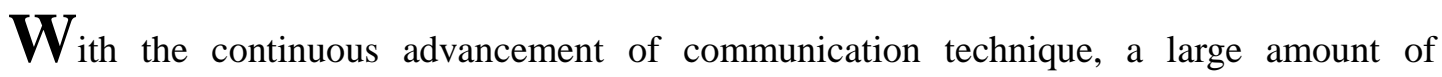
information is transmitted through the network, which improves work efficiency and brings convenience to people's lives. In the same way, this also leads criminals to easily steal information from others through the Internet. People enjoy the convenience brought by information technology and also bear the risk of disclosure of personal privacy information. Public key encryption technology has become an important means to achieve information security. In order to meet different needs, researchers have done much work to build specific public key encryption schemes in recent years.

In public key infrastructure (PKI), the user freely picks his/her own private key, then generates a public key and sends it to the certification authority (CA). CA generates a certificate to bind the user to his/her public key. A large amount of fees are used for the safekeeping, storage and transmission of certificates. To resolve the problem, Shamir [1] came up with identity-based cryptography. The user's sole personal information (email address, identity number, etc.) is his/her public key. Private key generator (PKG) yields the private key based on the public key and forwards it to the user. The information security of all users will be threatened if PKG is captured by an adversary. In 2003, Al-Riyami and Paterson [2] came up with certificateless cryptography. For one thing, the user picks a confidential value and yields a partial public key. For another, the user gets a partial private key, yielded by a key generation center (KGC) based on the identity information, through an authenticated channel.

\subsection{Related work}

Al-Riyami and Paterson [2] came up with the first CLE scheme. But, Libert and Quisquater [3] demonstrated that the scheme [2] is insecure, and put forward a means to construct CLE schemes with provably security. In 2010, Sun and Li [4] proposed a new CLE scheme with short-ciphertext, and proved it to be secure against chosen-ciphertext attacks (CCAs). In 2005, Baek et al. [5] presented a CLE scheme that does not require pairing operation. Sun et al. [6] indicated that the scheme [5] can achieve the security goals only in a weaker model, where Type I adversary is not allowed to change the user's public key. In 2013, Yan et al. [7] put forward a pairing-free CLE scheme and provided the security proofs in ROM. In same year, Guo et al. [8] brought forward a CLE scheme that does not require pairing operation. However, Deng et al. [9] pointed out that there are security flaws in scheme [8], then proposed a modified scheme. In 2018, Zhou et al. [10] came up with a CLE scheme that does not require pairing operation, and showed that it is secure against CCAs. In 2015, SK Hafizul et al. [11] put forward a certificateless multi-receiver encryption (CLMRE) scheme, and provided security proofs in ROM. In 2017, He et al. [12] proposed a pairing-free CLMRE scheme, which is efficient due to no Hash-to-Point (HTP) operation is required. In 
the same year, Gao et al. [13] brought forward a new CLMRE scheme, and proved that the receiver's identity information will not be leaked.

In 2007, Huang and Wong [14] came up with a common structure of CLE, which is provably secure in SM against the KGC attacks. In 2008, Dent et al. [15] presented a new CLE scheme, and asserted that it achieved confidentiality of the message in SM. But, Hwang et al. [16] indicated that the ciphertext indistinguishability against the KGC attacks does not hold for the scheme [15], then constructed a new CLE scheme. In 2009, Zhang and Wang [17] pointed out that the ciphertext indistinguishability against the key replacement attacks does not hold for the scheme [16], then constructed a new CLE scheme. However, Shen et al. [18] indicated that the ciphertext indistinguishability against the type II adversary does not hold for the scheme [17]. In 2014, Cheng et al. [19] evidenced that the ciphertext indistinguishability against the KGC attacks does not hold for the scheme [16], then proposed an improved scheme with provably security in SM. Reza et al. [20] put forward a common means to design CLE schemes with provably security in SM against CCAs, which come from a secure identity-based encryption scheme against chosen-plaintext attacks (CPAs).

\subsection{Motivations and contributions}

To increase security levels and reduce computing costs, researchers have proposed many CLE schemes. However, two problems remain in these schemes.

- Security proofs for most known CLE schemes are given in ROM

As we all know, the cryptography scheme provided with the security proofs in the ROM may be unsafe in a real situation. Therefore, these CLE schemes with provable security in ROM may be insecure in actual scenarios.

- High computation and storage costs

In the last ten years, scholars have proposed several concrete CLE schemes [15, 16, 17, 19], and tried to prove that they are secure in SM. However, there is only one scheme [19] that has been proven to be secure in SM. In these schemes [15, 16, 19], the size of the storage space required by the system is linearly related to the size of the user's identity information, and the times of addition operations on the elliptic curve group increases linearly with the size of the user's identity information. These increase the storage burden and computation cost for the users and the key generation center.

It is attractive to design an efficient CLE scheme and provide the security proofs in SM. We summarized the contributions as follows.

- We introduce the system model and security requirements of a CLE scheme in SM.

- We bring forward a new CLE scheme and offer the security proofs in SM. In order to get the hash function value, the adversary does not need to query the challenger, but directly calculates the hash function. 
- We give a comparison of the efficiency between three CLE schemes. In the new scheme, it was constant that the size of the storage space required by the system. It was constant that the number of three kinds of operations (addition, scalar multiplication, and pairing), so the computational cost is lower than other CLE schemes.

\subsection{Organization}

We introduce mathematical tools, system model and security requirements in Section 2, Section 3, and Section 4, respectively. We give a new CLE scheme and the security proofs in Section 5 and Section 6, respectively. We demonstrate an efficiency analysis of three CLE schemes in Section 7. We present some conclusions in Section 8.

\section{Preliminaries}

In this section, we introduce two mathematical tools: bilinear pairing and decisional bilinear Diffie-Hellman problem. Table 1 lists the notations used in the paper.

Table 1. Notations

\begin{tabular}{|c|c|}
\hline Symbol & Meaning \\
\hline$F_{p}$ & A prime finite field \\
\hline$q$ & A prime number \\
\hline$Z_{q}^{*}$ & A set of positive integers less than $q$. \\
\hline$\hat{e}: G_{1} \times G_{1} \rightarrow G_{2}$ & A bilinear pairing \\
\hline$P$ & A generator of the group $G_{1}$. \\
\hline$x, P_{p u b}$ & The master secret key and public key of system, $x \in Z_{q}^{*}$ and $P_{p u b}=x P$ \\
\hline$H_{1}, H_{2}, H_{3}$ & Three secure hash functions. \\
\hline$I D_{i}$ & The identity of $i^{\text {th }}$ user. \\
\hline$D_{i}$ & $\begin{array}{l}\text { The partial private key of } i^{\text {th }} \text { user, where } D_{i}=\left(R_{i}, d_{i}\right) \text {, } \\
\qquad R_{i}=r_{i} P, d_{i}=r_{i}+k_{i} X, k_{i}=H_{1}\left(I D_{i}, R_{i}\right)\end{array}$ \\
\hline
\end{tabular}




\begin{tabular}{|c|c|}
\hline$t_{i}$ & The secret value of $i^{\text {th }}$ user and $T_{i}=t_{i} P$. \\
\hline$P K_{i}$ & The public key of $i^{\text {th }}$ user, where $P K_{i}=\left(T_{i}, R_{i}\right)$. \\
\hline
\end{tabular}

\section{Bilinear pairing}

Let $\hat{e}: G_{1} \times G_{1} \rightarrow G_{2}$ be a mapping with the following attributes, where $G_{1}=(P)$ and $G_{2}$ are the additive and multiplicative groups of the $q$ order, respectively

- Bilinearity: $\hat{e}\left(a P_{1}, b P_{2}\right)=\hat{e}\left(P_{1}, P_{2}\right)^{a b}$ for all $P_{1}, P_{2} \in G_{1}$ and $a, b \in Z_{q}^{*}$.

- Non-degeneracy: There exist $P_{1}, P_{2} \in G_{1}$ such that $\hat{e}\left(P_{1}, P_{2}\right) \neq 1_{G_{2}}$.

- Computability: It is not difficult to compute $\hat{e}\left(P_{1}, P_{2}\right)$ for all $P_{1}, P_{2} \in G_{1}$.

Definition 1. Decisional bilinear Diffie-Hellman (DBDH) problem. Let $\hat{e}: G_{1} \times G_{1} \rightarrow G_{2}$ be a bilinear pairing. For $P \in G_{1}, \quad X \in G_{2}$, input a tuple $(P, a P, b P, c P, X)$, decide whether $X=\hat{e}(P, P)^{a b c}$.

\section{System Model}

A CLE scheme involves three distinct entities: key generation center (KGC), encryptor and decryptor, as shown in Fig. 1.

- KGC: It generates and publishes the system parameters. In addition, it yields a partial private key for the user.

- Encryptor: He encrypts a message to be a ciphertext by using the receiver's public key, then forwards that to the receiver.

- Decryptor: He obtains a message by decrypting the ciphertext with own private key.

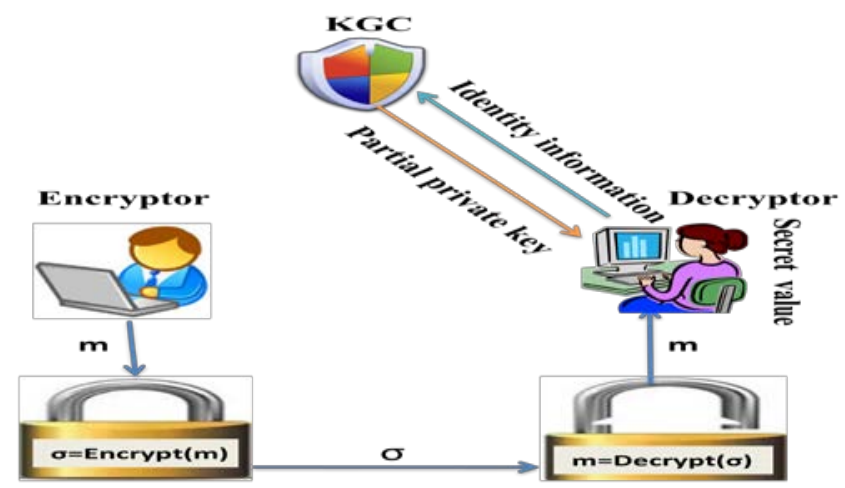

Fig. 1. Certificateless encryption 
A CLE scheme is constituted with the following six algorithms:

- Setup: Inputs a parameter $v$, KGC yields the msk (master secret key) and the params (system parameters).

- PPK-Extract: Inputs an identity $I D_{i} \in\{0,1\}^{*}$, KGC yields a partial private key $D_{i}$ and dispatches it to the user through a reliable channel..

- SV-Set: The user $I D_{i}$ picks a secret value $t_{i}$.

- UPK-Generate: The user $I D_{i}$ outputs his public key $P K_{i}$.

- Encrypt: Inputs a tuple $\left(m, I D_{i}, P K_{i}\right)$, the encryptor outputs a ciphertext $\sigma$.

- Decrypt: Inputs a tuple $\left(\sigma, I D_{i}, P K_{i}\right)$, the decryptor outputs a message $m$ or the symbol “0”.

\section{Security Requirements}

We described the security requirements in this section.

Definition 2. If the adversary's ascendency is insignificant in the coming two games, then the CLE scheme is indistinguishable (IND-CLE)

Game I. A challenger $\mathfrak{C}$ and a Type I adversary A play this game together.

Initialization. $\mathfrak{C}$ gets msk and params by implementing the Setup algorithm, maintains msk secret and forwards params to $A_{1}$

Phase 1. $A_{1}$ performs multiple types of queries.

- UPK-Query: $\mathfrak{c}$ outputs a user public key $P K_{i}$ when $A_{1}$ inputs an identity $I D_{i}$.

- UPK-Replacement: $\mathfrak{c}$ replaces $P K_{i}$ with $P K_{i}^{\prime}$ when $A_{1}$ inputs a tuple $\left(I D_{i}, P K_{i}^{\prime}\right)$,

- PPK-Query: $\mathfrak{C}$ outputs a partial public key $D_{i}$ when $A_{1}$ submits an identity $I D_{i}$. $\mathfrak{C}$ refuses to answer if the value $R_{i}$ has been replaced.

- SV-Query: $\mathfrak{C}$ outputs a secret value $t_{i}$ when $A_{1}$ inputs an identity $I D_{i}$. $\mathfrak{C}$ refuses to answer if the value $T_{i}$ has been replaced.

- ENC-Query: $\mathfrak{C}$ outputs a ciphertext $\sigma$ when $A_{1}$ submits a tuple $\left(m, I D_{i}, P K_{i}\right)$.

- DEC-Query: $\mathfrak{C}$ returns a message $m$ or the symbol " 0 " when $A_{1}$ submits a tuple $\left(\sigma, I D_{i}, P K_{i}\right)$.

Challenge. $A_{1}$ submits a tuple $\left(m_{0}, m_{1}, I D^{*}, P K^{*}\right)$, $\mathfrak{C}$ randomly selects a bit $\mu \in\{0,1\}$ and offers $A_{1}$ with $\sigma^{*}=\operatorname{Encrypt}\left(m_{\mu}, I D^{*}, P K^{*}\right)$. That fulfills the following conditions: 
1. $m_{0}$ and $m_{1}$ are two equal length messages.

2. $A_{1}$ did not make the PPK-Query for $I D^{*}$.

Phase 2. $A_{1}$ executes various queries again, which fulfills the following requirements.

1. $A_{1}$ did not make the PPK-Query for $I D^{*}$.

2. $A_{1}$ did not make the DEC-Query for $\sigma^{*}$.

Response. $A_{1}$ returns a bit $\mu^{\prime}$ and wins if $\mu^{\prime}=\mu$.

The advantage of $\quad A_{1}$ is defined as: $\operatorname{Ad} v_{A_{1}}^{I N D-C L E}=\left|\operatorname{Pr}\left[\mu^{\prime}=\mu\right]-\frac{1}{2}\right|$

Game II. A challenger $\mathfrak{C}$ and a Type II adversary $A_{2}$ play this game together.

Initialization. $\mathfrak{C}$ gets $m s k$ and params by implementing the Setup algorithm, then forwards them to $A_{2}$.

Phase 1. $A_{2}$ makes a series of queries as those in Game I.

Challenge. $A_{2}$ submits a tuple $\left(m_{0}, m_{1}, I D^{*}, P K^{*}\right)$, $\mathfrak{C}$ randomly selects a bit $\mu \in\{0,1\}$, provides $A_{2}$ with $\sigma^{*}=\operatorname{Encrypt}\left(m_{\mu}, I D^{*}, P K^{*}\right)$, which satisfy the following requirements.

1. $m_{0}$ and $m_{1}$ are two equal length messages.

2. $A_{2}$ did not perform SV-Query for $I D^{*}$.

3. $A_{2}$ did not perform UPK-Replacement for $T^{*}$.

Phase 2. $A_{2}$ executes various queries again, which satisfy the following requirements.

1. $A_{2}$ did not make SV-Query for $I D^{*}$.

2. $A_{2}$ did not perform UPK-Replacement for $T^{*}$.

3. $A_{2}$ did not make the DEC-Query for $\sigma^{*}$.

Response. $A_{2}$ returns a bit $\mu^{\prime}$ and wins if $\mu^{\prime}=\mu$.

The advantage of $A_{2}$ is defined as: $A d v_{A_{2}}^{I N D-C L E}=\left|\operatorname{Pr}\left[\mu^{\prime}=\mu\right]-\frac{1}{2}\right|$

\section{New scheme}

We constructed a new CLE scheme in this section. In the three schemes [15, 16, 19], the private key is generated based on each bit of the user's identity information. In our scheme, the identity information of the user is a whole, and the private key is generated based on the 
identity information, rather than directly related to each bit of the identity information. Our scheme is constituted with the following algorithms.

- Setup: Inputs a security parameter $v$, KGC does as follows.

1. Chooses two groups $G_{1}$ and $G_{2}$ with prime order $q>2^{v}$, a generator $P$ of $G_{1}$ and a bilinear pairing $\hat{e}: G_{1} \times G_{1} \rightarrow G_{2}$.

2. Selects three hash functions $H_{1}, H_{2}:\{0,1\}^{*} \rightarrow Z_{q}^{*}, H_{3}:\{0,1\}^{*} \rightarrow\{0,1\}^{l_{1}+l_{2}}$.

3. Sets the message space $M=\{0,1\}^{l_{1}}$.

4. Chooses a number $x \in Z_{q}^{*}$, computes $P_{p u b}=x P$ and sets $m s k=\{x\}$.

5. Publish params $=\left\{G_{1}, G_{2}, q, \hat{e}, P, P_{\text {pub }}, H_{1}, H_{2}, H_{3}\right\}$.

- PPK-Extract: Inputs an identity $I D_{i} \in\{0,1\}^{*}, \mathrm{KGC}$ randomly selects $r_{i} \in Z_{q}^{*}$ and computes $R_{i}=r_{i} P, k_{i}=H_{1}\left(I D_{i}, R_{i}\right), d_{i}=r_{i}+k_{i} x$, then sends $D_{i}=\left(R_{i}, d_{i}\right)$ to the user through an authenticated channel.

- SV-Set: The user $I D_{i}$ chooses at random $t_{i} \in Z_{q}^{*}$.

- UPK-Generate: The user $I D_{i}$ computes $T_{i}=t_{i} P$, and sets $P K_{i}=\left(T_{i}, R_{i}\right)$.

- Encrypt: To transmit a message $m \in M$ to Bob $\left(I D_{B} / P K_{B}\right)$, Alice carries out following steps:

1. Selects at random $u, v \in Z_{q}^{*}$, and $w \in\{0,1\}^{l_{2}}$.

2. Computes $U=u P, V=v P, \quad k_{B}=H_{1}\left(I D_{B}, R_{B}\right), \quad h=H_{2}\left(m, w, U, V, I D_{B}, P K_{B}\right)$,

$$
E=\hat{e}\left(u v P, R_{B}+k_{B} P_{p u b}+h T_{B}\right) \text { and } C=H_{3}(U, V, E, h) \oplus m \| w .
$$

3. Outputs the tuple $\sigma=(C, U, V, h)$.

- Decrypt: On receive the tuple $\sigma=(C, U, V, h)$, Bob carries out following steps:.

1. Computes $E=\hat{e}(U, V)^{d_{B}+h t_{B}}, \quad m \| w=H_{3}(U, V, E, h) \oplus C$. 
2. Checks whether $H_{2}\left(m, w, U, V, I D_{B}, P K_{B}\right)=h$. Accepts the message $m$ if the equality holds. Otherwise, rejects.

- On correctness

$$
\begin{aligned}
\hat{e}(U, V)^{d_{B}+h t_{B}} & =\hat{e}\left(\left(d_{B}+h t_{B}\right) U, V\right)=\hat{e}\left(\left(d_{B}+h t_{B}\right) u P, v P\right) \\
& =\hat{e}\left(u v P,\left(r_{B}+k_{B} X+h t_{B}\right) P\right)=\hat{e}\left(u v P, R_{B}+k_{B} P_{p u b}+h T_{B}\right)=E
\end{aligned}
$$

It is clear that the receiver can get the message by calling the decryption algorithm.

\section{Security of scheme}

We will give the security proofs in SM in this section. To obtain the hash function value, the adversary does not need to query the challenger, but directly calculates the hash function.

Theorem 1. Our scheme is indistinguishable against the Type I adversary in SM if the DBDH problem is hard.

Proof. Suppose that the tuple $(P, a P, b P, c P, X)$ is an example of DBDH problem. In order to determine whether $X=\hat{e}(P, P)^{a b c}, \mathfrak{C}$ will act as the challenger.

Initialization. Executing the Setup algorithm, c $\mathfrak{c}$ gets

$$
\text { params }=\left\{G_{1}, G_{2}, q, \hat{e}, P, P_{\text {pub }}=x P, H_{1}, H_{2}, H_{3}\right\} \text { and } m s k=\{x\}
$$

then sends the params to $A_{1}$.

Phase 1. Prior to other queries, an identity is first used for the public key queries. In order to store the query and answer, several initially empty lists are set.

- UPK-Query: $\mathfrak{C}$ maintains a list $L_{U}$ of tuple $\left(I D_{i}, t_{i}, r_{i}\right)$. When $A_{1}$ submits an identity $I D_{i}$, c does as follows.

Case 1. At the $j^{\text {th }}$ query, picks at random $t_{j} \in Z_{q}^{*}$, sets $I D_{j}=I D^{\diamond}, P K_{j}=P K^{\diamond}=\left(t_{j} P, a P\right)$, then adds the tuple $\left(I D_{j}, t_{j}, \diamond\right)$ to the list $L_{U}$.

Case 2. For $i \neq j$, c picks at random $t_{i}, r_{i} \in Z_{q}^{*}$ and returns $P K_{i}=\left(t_{i} P, r_{i} P\right)$, then adds the tuple $\left(I D_{i}, t_{i}, r_{i}\right)$ to the list $L_{U}$. 
- UPK-Replace: $\mathfrak{C}$ maintains a list $L_{R}$ of tuple $\left(I D_{i}, P K_{i}, P K_{i}^{\prime}\right)$. When $A_{1}$ submits a tuple $\left(I D_{i}, P K_{i}^{\prime}\right)$. C replaces $P K_{i}$ with $P K_{i}^{\prime}$ and adds $\left(I D_{i}, P K_{i}, P K_{i}^{\prime}\right)$ to the list $L_{R}$.

-PPK-Query: $\mathfrak{C}$ maintains a list $L_{D}$ of tuple $\left(I D_{i}, D_{i}\right)$. When $A_{1}$ submits an identity $I D_{i}$. If $I D_{i}=I D^{\diamond}$, $\mathfrak{c}$ fails and stops. Otherwise, $\mathfrak{c}$ finds $\left(I D_{i}, t_{i}, r_{i}\right)$ in the list $L_{U}$, outputs the $D_{i}$ by executing the PPK-Extract algorithm, then adds $\left(I D_{i}, D_{i}\right)$ to the list $L_{D}$.

- SV-Query: When $A_{1}$ submits an identity $I D_{i}$. $\mathfrak{C}$ finds $\left(I D_{i}, t_{i}, r_{i}\right)$ in the list $L_{U}$, returns $t_{i}$.

- ENC-Query: When $A_{1}$ inputs a tuple $\left(m, I D_{i}, P K_{i}\right)$, $\mathfrak{c}$ executes the Encrypt algorithm and returns a ciphertext $\sigma$.

- DEC-Query: When $A_{1}$ submits a tuple $\left(\sigma, I D_{i}, P K_{i}\right)$, $\mathfrak{c}$ carries out following steps.

1. $I D_{i} \neq I D^{\diamond}$ and $I D_{i} \notin L_{R}$, $\mathfrak{C}$ returns a message $m$ by calling the Decrypt algorithm.

2. $I D_{i} \in L_{R}$, then the $P K_{i}=\left(r_{i} P, t_{i} P\right)$ has been updated to $P K_{i}^{\prime}=\left(r_{i}^{\prime} P, t_{i}^{\prime} P\right)$. If $r_{i}^{\prime} \neq r_{i}$ (or $\left.t_{i}^{\prime} \neq t_{i}\right), \quad A_{1}$ must send $r_{i}^{\prime}$ (or $t_{i}^{\prime}$ ) to $\mathfrak{C}$, $\mathfrak{c}$ executes the Decrypt algorithm and returns a message $m$.

3. $I D_{i}=I D^{\diamond}$, c fails.

Challenge. $A_{1}$ submits a tuple $\left(m_{0}, m_{1}, I D^{*}, P K^{*}\right)$ satisfying the requirements in the Game I. If $I D^{*} \neq I D^{\diamond}$, $\mathfrak{c}$ aborts. Otherwise $I D^{*}=I D^{\diamond}=I D_{j}, \mathfrak{c}$ randomly selects a bit $\mu \in\{0,1\}$ and does as follows.

1. Finds $\left(I D_{j}, t_{j}, \diamond\right)$ in list $L_{U}$ and computes $k_{j}=H_{1}\left(I D_{J}, a P\right)$.

2. Picks at random $w \in\{0,1\}^{l_{2}}$, computes $h=H_{2}\left(m_{\mu}, w, b P, c P, I D^{*}, t_{j} P, a P\right)$.

3. Computes $E=X \cdot \hat{e}(b P, c P)^{k_{j} x+h t_{j}}$.

4. Computes $C=H_{3}(b P, c P, E, h) \oplus m_{\mu} \| w$. 
5. Outputs the ciphertext $\sigma^{*}=(C, b P, c P, h)$.

Phase 2. $A_{1}$ executes various queries again, which satisfy the terms in the Game I.

Response. $A_{1}$ outputs a bit $\mu^{\prime} \in\{0,1\}$.

Solve DBDH problem. $\mathfrak{c}$ returns “ 1 ” if $\mu^{\prime}=\mu$. Otherwise, returns “ 0 ”. If $X=\hat{e}(P, P)^{a b c}$, then

$$
\begin{aligned}
& E=X \cdot \hat{e}(b P, c P)^{k_{j} x+h t_{j}}=\hat{e}(P, P)^{a b c} \cdot \hat{e}\left(\left(k_{j} x+h t_{j}\right) b P, c P\right) \\
= & \hat{e}\left(\left(a+k_{j} x+h t_{j}\right) b P, c P\right)=\hat{e}\left(b c P,\left(a+k_{j} x+h t_{j}\right) P\right) \\
= & \hat{e}\left(b c P, R_{j}+k_{j} P_{p u b}+h T_{j}\right)
\end{aligned}
$$

Therefore, $\sigma^{*}$ is a valid ciphertext. Since $A_{1}$ has advantage $\varepsilon$. So

$$
\operatorname{Pr}\left[\mathfrak{C} \rightarrow 1 \mid X=\hat{e}(P, P)^{a b c}\right]=\operatorname{Pr}\left[\mu^{\prime}=\mu \mid X=\hat{e}(P, P)^{a b c}\right]=\frac{1}{2}+\varepsilon .
$$

If $X \neq \hat{e}(P, P)^{a b c}$, then $\sigma^{*}$ is an invalid ciphertext. Each part in $\sigma^{*}$ has the same distribution for $\mu=0$ and $\mu=1$.So $A_{1}$ has no superiority to differentiate the bit $\mu$. Hence

$$
\operatorname{Pr}\left[\mathfrak{C} \rightarrow 1 \mid X \neq \hat{e}(P, P)^{a b c}\right]=\operatorname{Pr}\left[\mu^{\prime}=\mu \mid X \neq \hat{e}(P, P)^{a b c}\right]=\frac{1}{2} .
$$

Probability. Let $q_{U}, q_{R}, q_{K}$ and $q_{D}$ be the number of UPK-Query, UPK-Replace, PPK-Query, and DEC-Query, respectively. Three events are indicated as follows.

$\pi_{1}: A_{1}$ did not perform UPK-Replacement for $R^{\diamond}$ and did not make the PPK-Query for $I D^{\diamond}$.

$\pi_{2}: \mathfrak{C}$ did not fail in decryption queries.

$\pi_{3}: I D^{*}=I D^{\diamond}$.

Obtaining the following results is not difficult.

$$
\begin{gathered}
\operatorname{Pr}\left[\pi_{1}\right]=\frac{q_{U}-q_{R}-q_{K}}{q_{U}} \cdot \operatorname{Pr}\left[\pi_{2} \mid \pi_{1}\right]=\left(1-\frac{1}{q_{U}}\right)^{q_{D}} \approx e^{-\frac{q_{D}}{q_{U}}} \cdot \operatorname{Pr}\left[\pi_{3} \mid \pi_{1} \wedge \pi_{2}\right]=\frac{1}{q_{U}-q_{R}-q_{K}} . \\
\operatorname{Pr}[\widetilde{\complement} \text { success }]=\operatorname{Pr}\left[\pi_{1} \wedge \pi_{2} \wedge \pi_{3}\right]=\operatorname{Pr}\left[\pi_{1}\right] \cdot \operatorname{Pr}\left[\pi_{2} \mid \pi_{1}\right] \cdot \operatorname{Pr}\left[\pi_{3} \mid \pi_{1} \wedge \pi_{2}\right] \\
=\frac{q_{U}-q_{R}-q_{K}}{q_{U}} \cdot e^{-\frac{q_{D}}{q_{U}}} \cdot \frac{1}{q_{U}-q_{R}-q_{K}} \approx \frac{1}{q_{U}} e^{-\frac{q_{D}}{q_{U}}}
\end{gathered}
$$


Therefore, if $A_{1}$ can win with the probability $\varepsilon$ in the Game I, then $\mathfrak{C}$ is able to decide whether $X=\hat{e}(P, P)^{a b c}$ with the probability $\frac{\varepsilon}{q_{U}} e^{-\frac{q_{D}}{q_{U}}}$.

Theorem 2. Our scheme is indistinguishable against the Type II adversary in SM if the DBDH problem is hard .

Proof. Suppose that the tuple $(P, a P, b P, c P, X)$ is an example of DBDH problem. In order to determine whether $X=\hat{e}(P, P)^{a b c}$, c will play the role of challenger..

Initialization. Executing the Setup algorithm, $\mathfrak{c}$ obtains

$$
\text { params }=\left\{G_{1}, G_{2}, q, \hat{e}, P, P_{\text {pub }}=x P, H_{1}, H_{2}, H_{3}\right\} \text { and } m s k=\{x\}
$$

and forwards them to $A_{2}$.

Phase 1.Prior to other queries, an identity is first used for public key queries. In order to store the query and answer, several initially empty lists are set.

- UPK-Query: $\mathfrak{c}$ safeguards a list $L_{U}$ of tuple $\left(I D_{i}, t_{i}, r_{i}\right)$. When $A_{2}$ inputs an identity $I D_{i}$, c does as follows.

Case 1. At the $j^{\text {th }}$ query, picks at random $r_{j} \in Z_{q}^{*}$, sets $I D_{j}=I D^{\diamond}$ and $P K_{j}=P K^{\diamond}=\left(a P, r_{j} P\right)$, then puts the tuple $\left(I D_{j}, \diamond, r_{j}\right)$ in the list $L_{U}$.

Case 2. For $i \neq j$, c randomly selects $t_{i}, r_{i} \in Z_{q}^{*}$ and returns $P K_{i}=\left(t_{i} P, r_{i} P\right)$, then puts the tuple $\left(I D_{i}, t_{i}, r_{i}\right)$ in the list $L_{U}$.

- UPK-Replacement: Same as that in Theorem 1.

- PPK-Query: $\mathfrak{C}$ safeguards a list $L_{D}$ of tuple $\left(I D_{i}, D_{i}\right)$. When $A_{2}$ submits an identity $I D_{i}$, $\mathfrak{C}$ finds $\left(I D_{i}, t_{i}, r_{i}\right)$ in list $L_{U}$, gives the $D_{i}$ by executing the PPK-Extract algorithm, then adds $\left(I D_{i}, D_{i}\right)$ to list $L_{D}$.

- SV-Query: When $A_{2}$ submits an identity $I D_{i}$. If $I D_{i}=I D^{\diamond}$, $\mathfrak{C}$ terminates the game. Otherwise, $\mathfrak{C}$ finds $\left(I D_{i}, t_{i}, r_{i}\right)$ in the list $L_{U}$, and returns $t_{i}$.

- ENC-Query, DEC-Query: Same as that in Theorem 1. 
Challenge. $A_{2}$ inputs a tuple $\left(m_{0}, m_{1}, I D^{*}, P K^{*}\right)$ satisfying the requirements in the Game II. If $I D^{*} \neq I D^{\diamond}$, c aborts. Otherwise $I D^{*}=I D^{\diamond}=I D_{j}$, $\mathfrak{c}$ picks a bit $\mu \in\{0,1\}$ and does as follows:

1. Finds $\left(I D_{j}, \diamond, r_{j}\right)$ in list $L_{U}$ and computes $k_{j}=H_{1}\left(I D_{j}, r_{j} P\right)$.

2. Randomly selects $w \in\{0,1\}^{l_{2}}$, computes $h=H_{2}\left(m_{\mu}, w, b P, c P, I D^{*}, a P, r_{j} P\right)$.

3. Computes $E=X^{h} \cdot \hat{e}(b P, c P)^{k_{j} x+r_{j}}$.

4. Computes $C=H_{3}(b P, c P, E, h) \oplus m_{\mu} \| w$.

5. Returns the ciphertext $\sigma^{*}=(C, b P, c P, h)$.

Phase 2. $A_{2}$ executes various queries again, which satisfy the terms in the Game II.

Response. $A_{2}$ outputs a bit $\mu^{\prime} \in\{0,1\}$.

Solve DBDH problem. $\mathfrak{c}$ outputs “ 1 ” if $\mu^{\prime}=\mu$. Otherwise, outputs “ 0 ”. If $X=\hat{e}(P, P)^{a b c}$, then

$$
\begin{aligned}
& E=X^{h} \cdot \hat{e}(b P, c P)^{k_{j} x+r_{j}}=\hat{e}(P, P)^{h a b c} \cdot \hat{e}\left(\left(k_{j} x+r_{j}\right) b P, c P\right) \\
& =\hat{e}\left(\left(h a+k_{j} x+r_{j}\right) b P, c P\right)=\hat{e}\left(b c P,\left(h a+k_{j} x+r_{j}\right) P\right) \\
& =\hat{e}\left(b c P, R_{j}+k_{j} P_{p u b}+h T_{j}\right)
\end{aligned}
$$

Therefore, $\sigma^{*}$ is a valid ciphertext. Since $A_{2}$ has advantage $\varepsilon$. So

$$
\operatorname{Pr}\left[\mathbb{C} \rightarrow 1 \mid X=\hat{e}(P, P)^{a b c}\right]=\operatorname{Pr}\left[\mu^{\prime}=\mu \mid X=\hat{e}(P, P)^{a b c}\right]=\frac{1}{2}+\varepsilon .
$$

If $X \neq \hat{e}(P, P)^{a b c}$, then $\sigma^{*}$ is a invalid ciphertext. Each part in $\sigma^{*}$ has the same distribution for $\mu=0$ and $\mu=1$. So $A_{2}$ has no superiority to differentiate the bit $\mu$. Hence

$$
\operatorname{Pr}\left[\mathfrak{C} \rightarrow 1 \mid X \neq \hat{e}(P, P)^{a b c}\right]=\operatorname{Pr}\left[\mu^{\prime}=\mu \mid X \neq \hat{e}(P, P)^{a b c}\right]=\frac{1}{2} .
$$

Probability. Let $q_{U}, q_{R}, q_{S}$ and $q_{D}$ be the number of UPK-Query, UPK-Replace, SV-Query, and DEC-Query, respectively. Three events are indicated as follows.

$\pi_{1}: A_{2}$ did not replace the value $T^{\diamond}$ and did not make the SV-Query for $I D^{\diamond}$. $\pi_{2}: \mathfrak{C}$ does not fail in decryption queries. 
$\pi_{3}: I D^{*}=I D^{\diamond}$.

It is not difficult to obtain the following results.

$$
\begin{gathered}
\operatorname{Pr}\left[\pi_{1}\right]=\frac{q_{U}-q_{R}-q_{S}}{q_{U}} \cdot \operatorname{Pr}\left[\pi_{2} \mid \pi_{1}\right]=\left(1-\frac{1}{q_{U}}\right)^{q_{D}} \approx e^{-\frac{q_{D}}{q_{U}}} \cdot \operatorname{Pr}\left[\pi_{3} \mid \pi_{1} \wedge \pi_{2}\right]=\frac{1}{q_{U}-q_{R}-q_{S}} . \\
\operatorname{Pr}\left[\mathbb{C}_{\text {success }}\right]=\operatorname{Pr}\left[\pi_{1} \wedge \pi_{2} \wedge \pi_{3}\right]=\operatorname{Pr}\left[\pi_{1}\right] \cdot \operatorname{Pr}\left[\pi_{2} \mid \pi_{1}\right] \cdot \operatorname{Pr}\left[\pi_{3} \mid \pi_{1} \wedge \pi_{2}\right] \\
=\frac{q_{U}-q_{R}-q_{S}}{q_{U}} \cdot e^{-\frac{q_{D}}{q_{U}}} \cdot \frac{1}{q_{U}-q_{R}-q_{S}} \approx \frac{1}{q_{U}} e^{-\frac{q_{D}}{q_{U}}}
\end{gathered}
$$

Therefore, if $A_{2}$ can win with the probability $\varepsilon$ in the Game II, then $\mathfrak{C}$ is able to decide whether $X=\hat{e}(P, P)^{a b c}$ with the probability $\frac{\varepsilon}{q_{U}} e^{-\frac{q_{D}}{q_{U}}}$

\section{Efficiency and comparison}

We give a contrast on the efficiency of the three CLE schemes in this section. Table 2 lists several notations used in this section.

Table 2. Notations

\begin{tabular}{|c|c|}
\hline Symbol & Meaning \\
\hline$B_{P}$ & A bilinear pairing operation. \\
\hline$S_{G_{1}}$ & A scale multiplication operation in $G_{1} \cdot$ \\
\hline$A_{G_{1}}$ & A addition operation in $G_{1} \cdot$ \\
\hline$M_{G_{2}}$ & A multiplication operation in $G_{2} \cdot$ \\
\hline$E_{G_{2}}$ & An exponentiation operation in $G_{2}$. \\
\hline$H$ & A general hash operation. \\
\hline$B_{I D}$ & The bit string of user's identity information, where $\left|B_{I D}\right|=n$ \\
\hline
\end{tabular}




\begin{tabular}{|c|c|}
\hline$B[i]_{I D}$ & The $i^{\text {th }}$ bit of user's identity information. \\
\hline$\Delta_{I D}$ & The set of indices $i$ such that $B[i]_{I D}=1$, namely $\Delta_{I D}=\left\{i: B[i]_{I D}=1\right\}$. \\
\hline$\left|G_{1}\right|$ & An element in $G_{1}$. \\
\hline$\left|G_{2}\right|$ & An element in $G_{2}$. \\
\hline$\left|Z_{q}^{*}\right|$ & An element in $Z_{q}^{*}$. \\
\hline
\end{tabular}

Table 3. Running time (in milliseconds)

\begin{tabular}{|c|c|c|c|c|c|}
\hline$B_{p}$ & $S_{G_{1}}$ & $A_{G_{1}}$ & $M_{G_{2}}$ & $E_{G_{2}}$ & $H$ \\
\hline 5.427 & 2.165 & 0.013 & 0.001 & 0.339 & 0.007 \\
\hline
\end{tabular}

For fairness and reasonableness, we analyze the three CLE schemes by using the third-party data. Implementing the basic cryptographic operations on a computer (with the Window 8 operating system, 4G bytes memory and an I5-4460S 2.90GHzprocessor), He et al. [21] acquired the running time, as shown in Table 3. To realize 1024-bit RSA security, they used a Tate pairing $\hat{e}: G_{1} \times G_{1} \rightarrow G_{2}$, where $G_{1}$ defined on a super singular curve $E / F_{p}$ : $y^{2}=x^{3}+1$ is an additive group of $q$ order, the lengths of $q$ and $p$ are 160 bits and 512 bits, separately.

Table 4. Comparison of three CLE schemes

\begin{tabular}{|c|c|c|c|}
\hline Scheme & Hwang [16] & Cheng [19] & New scheme \\
\hline \multirow{2}{*}{ Encrypt } & $n \cdot A_{G_{1}}+3 \cdot S_{G_{1}}$ & $n \cdot A_{G_{1}}+3 \cdot S_{G_{1}}$ & $B_{P}+2 \cdot A_{G_{1}}$ \\
& $+M_{G_{2}}+E_{G_{2}}+H$ & $+M_{G_{2}}+E_{G_{2}}+H$ & $+5 \cdot S_{G_{1}}+3 \cdot H$ \\
\hline \multirow{2}{*}{ Decrypt } & $4 \cdot B_{P}+(n+2) \cdot A_{G_{1}}$ & $4 \cdot B_{P}+(n+2) \cdot A_{G_{1}}$ & \\
& $+2 \cdot M_{G_{2}}+H$ & $+3 \cdot M_{G_{2}}+H$ & $B_{P}+S_{G_{1}}+2 \cdot H$ \\
\hline
\end{tabular}




\begin{tabular}{|c|c|c|c|}
\hline Time $(n=60)$ & $\begin{array}{c}0.026 n+28.585 \\
(30.145)\end{array}$ & $\begin{array}{c}0.026 n+28.586 \\
(30.146)\end{array}$ & 23.905 \\
\hline $\begin{array}{c}\text { Size of Params } \\
(n=60)\end{array}$ & $(2 n+4)\left|G_{1}\right|+\left|G_{2}\right|$ & $(2 n+4)\left|G_{1}\right|+\left|G_{2}\right|$ & $2\left|G_{1}\right|(128$ bytes $)$ \\
\hline $\begin{array}{c}\text { Size of Msk } \\
(n=60)\end{array}$ & $(2 n+4)\left|Z_{q}^{*}\right|$ & $(2 n+4)\left|Z_{q}^{*}\right|$ & $\left|Z_{q}^{*}\right|(20$ bytes $)$ \\
\hline Security & $(2480$ bytes $)$ & $(2480$ bytes $)$ & Yes \\
\hline
\end{tabular}

For reasonableness, we suppose that the size of the user's identity information is 60 bits, i.e. $n=60$. It is a reasonable assumption that $\left|\Delta_{I D}\right|=\left\lfloor\frac{n}{2}\right\rfloor$. We use an intuitive way to evaluate the calculation cost. In [16], encrypting a plaintext demands $n$ addition operations in $G_{1}, 3$ scale multiplication operations in $G_{1}, 1$ multiplication operation in $G_{2}, 1$ exponentiation operation in $G_{2}$ and 1 general hash operation. Decryption a ciphertext requires 4 bilinear pairing operations, $n+2$ addition operations in $G_{1}, 2$ multiplication operations in $G_{2}$ and 1 general hash operation. So the resulting running time is

$$
4 \times 5.427+(2 \times 60+2) \times 0.013+3 \times 2.165+3 \times 0.001+0.339+2 \times 0.007=30.145 \mathrm{~ms} .
$$

In [19], encrypting a plaintext demands $n$ addition operations in $G_{1}, 3$ scale multiplication operations in $G_{1}, 1$ multiplication operation in $G_{2}, 1$ exponentiation operation in $G_{2}$ and 1 general hash operation. Decrypting a ciphertext requires 4 bilinear pairing operations, $n+2$ addition operations in $G_{1}, 3$ multiplication operations in $G_{2}$ and 1 general hash operation. So the resulting running time is

$$
4 \times 5.427+(2 \times 60+2) \times 0.013+3 \times 2.165+4 \times 0.001+0.339+2 \times 0.007=30.146 \mathrm{~ms} .
$$

In the new scheme, encrypting a plaintext demands 1 bilinear pairing operation, 2 addition operations in $G_{1}$, 5 scale multiplication operations in $G_{1}$ and 3 general hash operations. Decrypting a ciphertext requires 1 bilinear pairing operation, 1 scale multiplication operations in $G_{1}$ and 2 general hash operations. So the resulting running time is

$$
2 \times 5.427+2 \times 0.013+6 \times 2.165+5 \times 0.007=23.905 \mathrm{~ms} .
$$

Table 4 and Fig. 2 illustrate the computation costs of three different CLE schemes. 


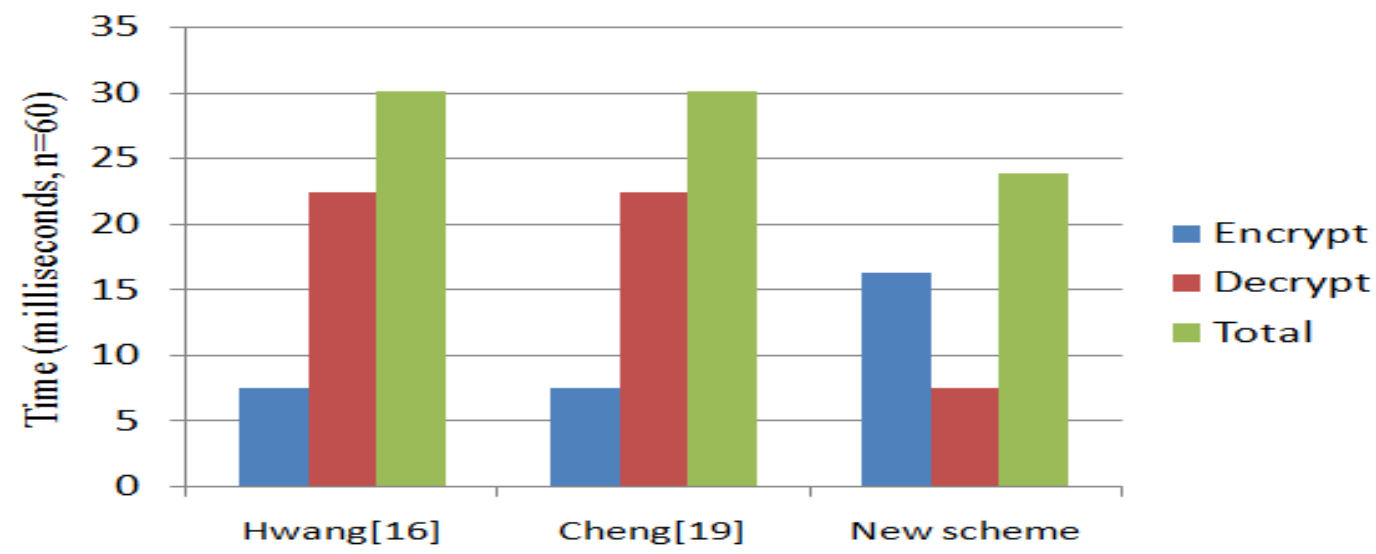

Fig. 2. Computation cost

Next, we evaluated the size of params and msk. In two schemes [16, 19], the params contain $2 n+5$ points over a elliptic curve $E / F_{p}: y^{2}=x^{3}+1$, thus the size is $\frac{(2 \times 60+5) \times 512}{8}=8000$ bytes. The $m s k$ contains $2 n+4$ points in $Z_{q}^{*}$, thus the size is $\frac{(2 \times 60+4) \times 160}{8}=2480$ bytes. In the new scheme, the params contain only 2 points over a elliptic curve $E / F_{p}: y^{2}=x^{3}+1$, thus the size is $\frac{2 \times 512}{8}=128$ bytes. The msk contains only one point in $Z_{q}^{*}$, thus the size is $\frac{160}{8}=20$ bytes. Table 4 and Fig. 3 illustrate the storage costs of the three CLE schemes.

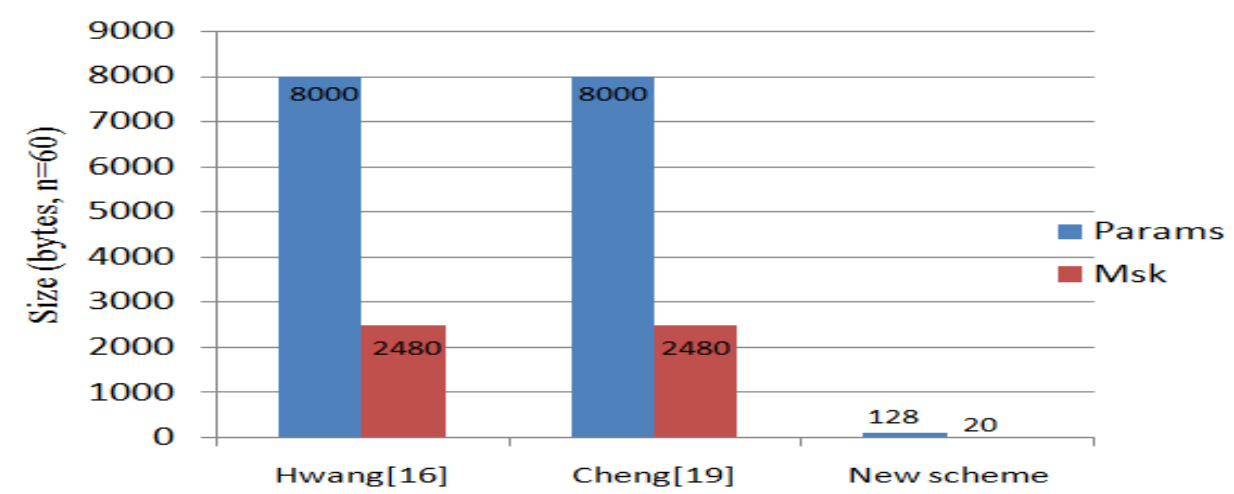

Fig. 3. Storage expenses 


\section{Conclusion}

Certificateless cryptography is a significant technique to achieve data security and personal privacy protection. The security proofs of most known CLE schemes are done in ROM. It is well known, a cryptography scheme is not necessarily safe in real situations, even if its security proofs have been completed in ROM. There is only one CLE scheme [19], whose security proofs are completed in SM. But, the size of the storage space required by the system is linearly related to the size of the user's identity information. That adds to the storage burden of the key generation center. In this paper, we construct a fresh CLE scheme and complete the security proofs in SM. In the new scheme, it was constant that the size of the storage space required by the system, it was constant that the number of three kinds of operations (addition, scalar multiplication, and pairing). The computation cost and storage cost of the new scheme are lower than that of the previous ones.

\section{Acknowledgment}

The authors are grateful to the anonymous referees for their helpful comments and insightful suggestions. This research is supported by the National Natural Science Foundation of China under Grant No. 61962011, the Innovation Group Major Research Projects of Department of Education of Guizhou Province under Grant No.KY[2016]026. the Guizhou Provincial Science and Technology Foundation under Grant No.[2019]1434.

\section{References}

[1] Shamir, A., "Identity-based cryptosystem and signature scheme,” Advances in Cryptology-Crypto, LNCS, vol.196, pp. 47-53, 1984. Article (CrossRef Link)

[2] Al-Riyami, S.S., and Paterson, K.G., “Certificateless public key cryptography,” Advances in Cryptology-Asiacrypt, LNCS, vol.2894, pp.452-473, 2003. Article (CrossRef Link)

[3] Libert, B., and Quisquater, J., “On constructing certificateless cryptosystems from identity based encryption,” in Proc. of International Workshop on Public Key Cryptography, LNCS, vol.3958, pp.474-490, 2006. Article (CrossRef Link)

[4] Sun, Y., and Li, H., "short-ciphertext and BDH-based CCA2 secure certificateless encryption," Science China: Information Science, vol.53, pp.2005-2015, 2010. Article (CrossRef Link)

[5] Baek, J., Safavi-Naini, R., and Susilo, W., “Certificateless public key encryption without pairing," in Proc. of International Conference on Information Security, LNCS, vol.3650, pp.134-148, 2005. Article (CrossRef Link)

[6] Sun, Y., Zhang, F., and Baek, J., "Strongly secure certificateless public key encryption without pairing," in Proc. of International Conference on Cryptology and Network Security, LNCS, vol.4856, pp.194-208, 2007. Article (CrossRef Link) 
[7] Yan, X., Gong, P., Bai, Z., Wang, J., and Li, P., "New certificateless public key encryption scheme without pairing,” IET Information Security, vol.7, iss.4, pp.271-276, 2013. Article (CrossRef Link)

[8] Guo, R., Wen, Q., Shi, H., Jin, Z., and Zhang, H, “An efficient and provably secure certificateless public key encryption scheme for telecare medicine information systems,” Journal of Medical Systems, vol.37, no.5, pp.9965, 2013. Article (CrossRef Link)

[9] Deng, L., Zeng, J., Wang, X, “An improved certificateless encryption scheme for telecare medicine information systems,” Journal of Internet Technology, vol.18, no.2, pp.223-227, 2017. Article (CrossRef Link)

[10] Zhou, Y., and Yang, B., “Leakage-resilient CCA2-secure certificateless public-key encryption scheme without bilinear pairing,” Information Processing Letters, vol.130, pp.16-24, 2018. Article (CrossRef Link)

[11] SK Hafizul, I., Muhammad, K., and Ali M, Al., “Anonymous and provably secure certificateless multi receiver encryption without bilinear pairing," Security and Communication Networks, vol.8, pp.2214-2231, 2015. Article (CrossRef Link)

[12] He, D., Wang, H., Wang, L., Shen, J., and Yang, X., "Efficient certificateless anonymous multi-receiver encryption scheme for mobile devices,” Soft Computing, vol.21, no.22, pp.6801-6810, 2017. Article (CrossRef Link)

[13] Gao, R., Zeng, J., and Deng L., "Efficient certificateless anonymous multi-Receiver encryption scheme without bilinear parings,” Mathematical Problems in Engineering, Article ID 1486437, 13 pages, 2018. Article (CrossRef Link)

[14] Huang, Q., and Wong, D.S., “Generic certificateless encryption in the standard model,” in Proc. of International Workshop on Security, LNCS, vol.4752, pp.278-291, 2007.

Article (CrossRef Link)

[15] Dent, A.W., Libert, B., and Paterson, K.G., "Certificateless encryption schemes strongly secure in the standard model,” in Proc. of International Workshop on Public Key Cryptography, LNCS, vol.4939, pp.344-359, 2008. Article (CrossRef Link)

[16] Hwang, Y.H., Liu, J.K., and Chow, S.S, “Certificateless public key encryption secure against malicious KGC attacks in the standard model,” Journal of Universal Computer Science, vol.14, no.3, pp.463-480, 2008. Article (CrossRef Link)

[17] Zhang, G., and Wang, X, “Certificateless encryption scheme secure in standard model,” Tsinghua Science \& Technology, vol.14, no.4, pp.452-459, 2009. Article (CrossRef Link)

[18] Shen, L., Zhang, F., Sun, Y., and Li, S., “Cryptanalysis of a certificateless encryption scheme in the standard model," in Proc. of International Conference on Intelligent Networking and Collaborative Systems, pp.329-333, 2012. Article (CrossRef Link)

[19] Cheng, L., Wen, Q., Jin, Z., and Zhang, H., “Cryptanalysis and improvement of a certificateless encryption scheme in the standard model,” Frontiers of Computer Science, vol.8, no.1, pp.163-173, 2014. Article (CrossRef Link) 
[20] Reza, S., Ron S., and Josef, Pieprzyk., "Lattice-based certificateless public-key encryption in the standard model,” International Journal of Information Security, vol.13, pp.315-333, 2014. Article (CrossRef Link)

[21] He, D., Zeadally, S., Kumar, N., and Wu, W., "Efficient and anonymous mobile user authentication protocol using self-certified public key cryptography for multi-server architectures," IEEE transactions on information forensics and security, vol.11, no.9, pp.2052-2064, 2016. Article (CrossRef Link)

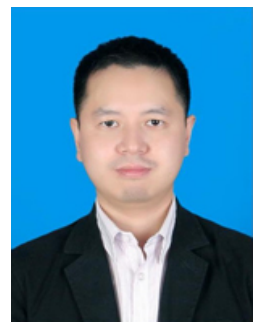

Lunzhi Deng received his B.S. from Guizhou Normal University, Guiyang, PR China, in 2002; M.S. from Guizhou Normal University, Guiyang, PR China, in 2008; and Ph.D. from Xiamen University, Xiamen, PR China, in 2012. He is now a professor in the School of Mathematical Sciences, Guizhou Normal University, Guiyang, PR China. His recent research interests include cryptography and information safety.

Email:denglunzhi@163.com

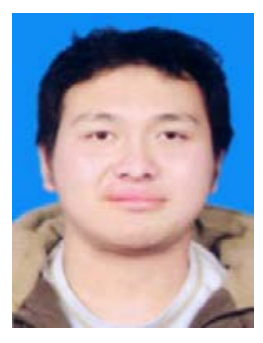

Tian Xia received his B.S. from Guizhou normal University, PR China, in 2009; he is now a graduate student at Guizhou Normal University in China. His recent research interests include digital signature and encryption protocols.

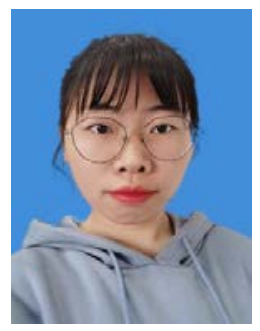

Xiuru He received her B.S. from Huaibei normal University, PR China, in 2018; She is now a graduate student at Guizhou Normal University in China. Her recent research interests include authentication protocol and information safety. 